\title{
Arousal frequency is associated with increased fatigue in obstructive sleep apnea
}

\author{
Herbert J. Yue • Wayne Bardwell • Sonia Ancoli-Israel • \\ José S. Loredo • Joel E. Dimsdale
}

Received: 1 December 2008 /Revised: 18 February 2009/Accepted: 2 March 2009/Published online: 25 March 2009

(C) The Author(s) 2009. This article is published with open access at Springerlink.com

\begin{abstract}
Objective Fatigue is an important and often underemphasized symptom in patients with obstructive sleep apnea (OSA). Sleep fragmentation, i.e., arousals and disruptions in sleep architecture, is common in patients with OSA and may potentially contribute to their fatigue. We hypothesized that arousal frequency and changes in sleep architecture contribute to the fatigue experienced by patients with OSA. Design Seventy-three patients with diagnosed but untreated OSA (AHI $\geq 15$ ) were enrolled in this study. A baseline polysomnogram was obtained, and fatigue was measured with the Multidimensional Fatigue Symptom Inventoryshort form (MFSI-sf). We evaluated the association between
\end{abstract}

\footnotetext{
H. J. Yue · J. S. Loredo

Department of Medicine, University of California,

San Diego School of Medicine,

La Jolla, CA, USA

W. Bardwell · S. Ancoli-Israel · J. E. Dimsdale Department of Psychiatry, University of California,

San Diego School of Medicine,

La Jolla, CA, USA

W. Bardwell · J. E. Dimsdale

Moores Cancer Center, UCSD Medical Center,

La Jolla, CA, USA

S. Ancoli-Israel

Veterans Affairs San Diego Healthcare System,

San Diego, CA, USA

H. J. Yue $(\bowtie)$

Department of Pulm/Crit Care Medicine,

UC San Diego Medical Center,

200 West Arbor Drive,

San Diego, CA 92103-8383, USA

e-mail: hyue@ucsd.edu
}

fatigue and arousals and various polysomongraphic variables, including sleep stages and sleep efficiency.

Results Significant correlations between MFSI-sf subscale scores and various arousal indices were noted. Emotional fatigue scores were associated with total arousal index $(r=$ $0.416, p=.021)$, respiratory movement arousal index $(r=$ $0.346, p=.025)$, and spontaneous movement arousal index $(r=0.378, p=.025)$. Physical fatigue scores were associated with total arousal index $(r=0.360, p=.033)$ and respiratory movement arousal index $(r=0.304, p=.040)$. Percent of stage 1 sleep and REM sleep were also associated with physical and emotional fatigue scores. Hierarchal linear regression analysis demonstrated that emotional fatigue scores were independently associated with spontaneous movement arousals after controlling for age, body mass index, depression, and sleep apnea severity.

Conclusions These findings suggest that arousals may contribute to the fatigue seen in patients with OSA.

Keywords Obstructive sleep apnea $\cdot$ Fatigue .

Sleep architecture $\cdot$ Arousals

\section{Introduction}

Obstructive sleep apnea (OSA) is a common condition found in approximately $10 \%$ of the general population [1]. It is characterized by episodes of upper airway obstruction during sleep, with resultant apneas and hypopneas, frequent arousals from sleep, and repetitive transient drops in oxyhemoglobin saturation. Patients with OSA typically have symptoms of excessive daytime sleepiness (EDS), insomnia, and fatigue [2].

There is less known about the incidence and impact of fatigue in patients with OSA. Fatigue as a concept is more difficult to define, and sometimes, it is used by patients 
interchangeably with sleepiness. Nonetheless, correlation studies have demonstrated poor agreement between measures of sleepiness and fatigue, suggesting that fatigue is a distinct entity in these patients [3-5]. Current ideas of fatigue include diverse conceptualizations referring to acute vs. chronic fatigue, physiological vs. psychological fatigue, or central vs. peripheral fatigue [6, 7]. In OSA, patient fatigue has not been well characterized. Fatigue is a common problem, with a reported incidence of $24 \%$ in the general US population, rising up to $50-90 \%$ in patients with cancer, chronic lung disease, or multiple sclerosis [810]. Fatigue is associated with significant morbidity; for instance, cancer patients report that fatigue is their most troubling symptom [11].

There have been limited studies looking at fatigue as an independent symptom in OSA. Although EDS is the most prominent reason for referral in OSA patients, several studies have found that the incidence of self-reported fatigue surpasses that of sleepiness in these patients [3, 4]. When patients with OSA were asked to report their most prominent symptom, more patients chose fatigue over sleepiness $(40 \%$ vs. $22 \%$ ) [4]. When OSA is successfully treated, patients reported improvements in their fatigue $[12,13]$.

It is not clear what drives the feeling of fatigue in apneics. Measures of apnea severity, such as the apnea hypopnea index (AHI) or various measures of oxygen desaturation, are not correlated with fatigue measurements [3-5, 14-16]. In studies of chronic fatigue syndrome, decreased amounts of slow wave sleep and sleep efficiency have been associated with fatigue, although the results have been inconsistent [17, 18]. There may be subtle ethnic differences as well differences in the association of fatigue and sleep; previous work has demonstrated that decreases in slow wave sleep are associated with increased fatigue scores particularly in African-American subjects [19]. In normal adults subjected to partial sleep deprivation, increases in fatigue scores were associated with increases in stages 1 and 2 sleep [20]. There is no consensus, however, regarding which polysomnographic changes are consistently associated with fatigue, and the literature on fatigue in patients with OSA is limited.

There has also been little investigation examining EEG arousals and fatigue. Patients with OSA experience frequent EEG arousals, defined as abrupt changes in EEG frequency to alpha or theta without spindle activity. Arousals can also be further classified into cortical, movement, and respiratory arousals [21]. These frequent arousals may potentially contribute to increased fatigue through sleep fragmentation. Prior work in models of upper airway resistance syndrome found that arousal frequency was associated with increased fatigue [22].

In summary, fatigue appears to be understudied in OSA patients, despite its being a prominent and frequent symptom in this population. Prior studies have demonstrated no clear association of fatigue measurements with apnea severity. Little has been done looking at the association of fatigue with polysomnographic disturbances such as sleep stage changes and sleep fragmentation in OSA. This study examines the contribution of arousal frequency to fatigue scores, after controlling for a number of factors that would likely influence fatigue. We hypothesize that arousal frequency as well as changes in sleep architecture contribute to the fatigue experienced by patients with OSA.

\section{Methods}

Patients Participants with known or suspected obstructive sleep apnea not previously treated were recruited by advertisement or word of mouth. Enrolled patients underwent a history and physical examination as well as answered questions about their medical history to determine eligibility for the study. Patients with a history of kidney, liver, pulmonary or heart disease, stroke, or other neurologic disorders were excluded from participation. Women were also excluded if pregnant. Basic intake data were obtained on all participants, including age, ethnicity, vital signs, weight and body mass index, education, and occupation. After a baseline polysomnogram, 73 (59 men and 14 women) had OSA (AHI $\geq 15)$ and were invited to participate in the study. Study participants also completed the Center for Epidemiologic Studies-Depression scale (CES-D) and Multidimensional Fatigue Symptom Inventory-short form (MFSI-sf) to evaluate the extent of depressive and fatigue symptoms. The study was approved by the Institutional Review Board at the University of California, San Diego (UCSD), and participants were studied after obtaining written informed consent.

CES-D The CES-D is a commonly used tool for measuring the prevalence of depressive symptoms in a population [23]. It measures current levels of depressive symptoms across 20 questions that inquire about symptoms such as hopelessness, appetite loss, depressed mood, feelings of guilt, sleep disturbance, and psychomotor retardation. Respondents report how often they have experienced depressive symptoms over the past week: $0=$ rarely or none of the time. $1=$ some or a little of the time, 2 occasionally or a moderate amount of time, and $3=$ most or all of the time. Instruments are scored with a raw sum, with scores $\geq 16$ suggesting an increased risk of depression. The CES-D has been validated across a number of different patient populations and has been used to determine the influence of depression on health outcomes. 
MFSI-sf The MFSI-sf is a 30 -item instrument designed to assess global, somatic, affective, cognitive, and behavioral symptoms of fatigue [24]. Respondents report how often they have experienced symptoms of fatigue over the prior week, with $0=$ not at all to $4=$ extremely. Scores are summed to obtain subscale scores, which include general fatigue, physical fatigue, emotional fatigue, mental fatigue, and vigor. The vigor subscale score is then subtracted from the sum of the four fatigue subscales to obtain a total fatigue score. The range of scores for each subscale ranges from 0 to 24 and for the MFSI-total score, -24 to 96 . Higher scores indicate increased fatigue. The instrument has been used extensively in different patient populations to assess for the prevalence and severity of fatigue [25].

Polysomnography All participants were admitted to the Gillin Laboratory of Sleep and Chronobiology of the UCSD General Clinical Research Center and underwent baseline overnight polysomnography on one night as previously described [21]. Polysomnograms were always recorded as part of this study at the onset of enrollment. Electroencephalography, electroculography, chin electromyography, and thoracic and abdominal respiratory effort were recorded on a Grass Heritage digital polysomnograph (Model PSG36-2, Astro-Med, Inc, West Warwick, RI, USA). Oxygen saturation was measured with a pulse oximeter (Biox 3740; Ohmeda: Louisville, CO, USA). Sleep recordings were scored in 30-s epochs and staged according to the criteria of Rechtshaffen and Kales [26]. Percent of total sleep time for each sleep stage (1, 2, slow wave sleep, and REM), AHI, oxygen desaturation index (ODI), and arousal indices were calculated for each participant. Apneas and hypopneas were defined as decrements in airflow of $>90 \%$ and $>50 \%$ but $<90 \%$ from baseline lasting at least $10 \mathrm{~s}$, respectively. The number of apneas and hypopneas per hour of sleep were calculated to obtain the AHI. Arousals were scored according to the general criteria of the ASDA and globally defined as sudden increases in EEG frequency to alpha or theta without spindle activity, lasting at least $3 \mathrm{~s}$ but less than $15 \mathrm{~s}$, with at least $10 \mathrm{~s}$ of sleep recorded prior to the arousal [27]. Arousals were then further subclassified as previously described into the following categories [21]:

1. Total arousals: the sum of all cortical, movement, and snore arousals divided by the total sleep time.

2. Snore arousal: any type of arousal occurring immediately following a period of crescendo snoring in the absence of an apneic event. Snore arousals include respiratory effort related arousals.

3. Limb movement arousal: sudden increase in EEG frequency following a spontaneous limb movement in the absence of an apneic event or crescendo snoring.
4. Periodic limb movement arousal: sudden increase in EEG frequency following a periodic limb movement in the absence of an apneic event or crescendo snoring.

5. Respiratory cortical arousal: sudden increase in EEG frequency without obvious rise in chin or leg EMG activity occurring within three breaths after the termination of a respiratory apneic event.

6. Respiratory movement arousal: sudden increase in EEG frequency associated with increased in chin or of the tibialis anterior muscle EMG activity of the chin or of the tibilias anterior muscle occurring within three breaths after the termination of a respiratory apneic event.

7. Spontaneous cortical arousal: sudden increase in EEG frequency without an increase in EMG activity occurring in the absence of an obvious precipitating event.

8. Spontaneous movement arousal: sudden increase in EEG frequency associated with an increase in chin or of the tibialis anterior muscle EMG activity of the chin or of the tibiliais anterior muscle, occurring in the absence of an obvious precipitating event.

Indices for each arousal type were calculated by summing the number of each particular arousal and dividing per hour of the total sleep time. Scoring reliability measures for measures for arousal indices have been described previously [28].

Statistical analyses Descriptive statistics were determined for all demographic and sleep architecture characteristics. Distributions for the arousal indices were square root transformed to approximate a normal distribution. Pearson correlations were performed between MFSI-sf subscale scores and sleep architecture measures as well as the various arousal indices ${ }^{1}$. In order to account for multiple comparisons due to the multiple fatigue and sleep variables, we applied Fisher's Least Significant Difference test [29]. This is a two-stage test where at the first stage, the grand null hypothesis encompassing all the hypotheses of interest is tested. If this grand null is rejected, then the second stage tests the individual hypotheses separately. To this end, we first performed a multivariate ANOVA (MANOVA) using MFSI-sf subscales as dependent variables with sleep parameters (i.e., percent of stages of sleep, sleep efficiency, arousals, etc.) as covariates. If the $F$ test for a MANOVA model was statistically significant, we proceeded to the second stage and tested individual hypotheses. We then

\footnotetext{
${ }^{1}$ We did not evaluate associations between MFSI-sf general fatigue subscale and measures of sleep architecture or arousals because as noted in the literature ${ }^{28}$ and as found in our preliminary analyses, the MFSI-sf general fatigue scores are so tightly correlated with MFSI-sf total scores $(\mathrm{r}=.921, \mathrm{p}<.001)$ that little is to be gained by analysis of both of these measures in the face of their colinearity.
} 
performed separate hierarchical regression analyses to examine arousal relationships with the fatigue measures to determine if sleep architecture measures or arousals contributed additional independent information to fatigue severity, after controlling for demographic variables, depression, and sleep apnea severity. These hierarchical regressions were employed to examine fatigue/arousal relationships only where a significant association was revealed on MANOVA analysis. Each MFSI-sf subscale was the dependent variable. At step 1, we forced entry of age and BMI. For step 2, we entered total CESD scores. For step 3, we entered AHI and the oxygen desaturation index. For step 4, we entered the pertinent arousal index and/or sleep architecture measure. For fatigue subscales in which total arousals and respiratory movement arousals both had significant MANOVA statistics, we entered only respiratory movement arousals in step 4, because total arousals and respiratory movement arousals were highly correlated with each other $(r=0.966, p \leq 0.001)$ Statistics were considered significant at $p<0.05$. Statistical analyses were performed using SPSS statistical software (SPSS for Windows 12.0: SPSS Inc.; Chicago, IL, USA).

\section{Results}

Seventy-three patients with obstructive sleep apnea completed the study. Table 1 describes subjects' demographic and anthropometric characteristics. Patients were on average middle aged (50 years old \pm 9 years), predominantly male, and mildly obese $\left(B M I=31.1 \pm 6.3 \mathrm{~kg} / \mathrm{m}^{2}\right)$. Average CES-D scores were somewhat elevated at $11.1 \pm 9.0$. Mean total MFSI-sf scores were elevated at $8.8 \pm 17.7$, suggestive of moderate fatigue [24, 25].

Table 1 Patient characteristics

\begin{tabular}{ll}
\hline Characteristic & Mean \pm SD \\
\hline Age, years & $49.3 \pm 9.2$ \\
Gender & 59 male, 14 female \\
BMI, kg/m² & $31.1 \pm 6.3$ \\
CESD & $11.1 \pm 9.0$ \\
MFSI-sf Total Score & $8.8 \pm 17.7$ \\
MFSI-sf General Fatigue & $7.9 \pm 5.9$ \\
MFSI-sf Physical Fatigue & $3.5 \pm 4.0$ \\
MFSI-sf Emotional Fatigue & $4.2 \pm 3.7$ \\
MFSI-sf Mental Fatigue & $5.3 \pm 3.9$ \\
MFSI-sf Vigor & $12.1 \pm 5.0$ \\
\hline
\end{tabular}

CESD Center for Epidemologic Studies-Depression Scale, MFSI-sf multidimensional fatigue symptom inventory-short form
Table 2 Sleep characteristics $(n=73)$

\begin{tabular}{lc}
\hline Sleep recording measures & Mean \pm SD \\
\hline Stage 1 sleep, \% & $16.9 \pm 10.3$ \\
Stage 2 sleep, \% & $61.0 \pm 9.3$ \\
Slow wave sleep (stage 3 and 4), \% & $2.8 \pm 5.0$ \\
REM sleep, \% & $16.9 \pm 6.2$ \\
Sleep efficiency, \% & $82.9 \pm 8.5$ \\
Wake after sleep onset, min & $59.4 \pm 32.3$ \\
AHI & $49.3 \pm 29.7$ \\
ODI & $10.8 \pm 20.9$ \\
Total arousal index & $33.2 \pm 26.0$ \\
Snore arousal index & $2.1 \pm 2.2$ \\
Spontaneous cortical arousal index & $0.7 \pm 1.0$ \\
Respiratory cortical arousal index & $1.7 \pm 2.7$ \\
Spontaneous movement arousal index & $1.6 \pm 2.1$ \\
Respiratory movement arousal index & $26.3 \pm 27.5$ \\
Limb movement arousal index & $0.3 \pm 0.4$ \\
Periodic limb movement index & $0.3 \pm 0.4$ \\
\hline
\end{tabular}

Table 2 depicts the baseline sleep parameters. On average, the study population had severe OSA (AHI= $49.3 \pm 29.7)$. The mean total arousal index was also significantly elevated at $33.2 \pm 26.0$. The majority of the arousals consisted of respiratory movement arousals. Stages 1 and 2 sleep were high at $16.9 \%$ and $61.0 \%$, respectively, and slow wave and REM sleep were both low at $2.8 \%$ and $16.9 \%$, respectively; sleep efficiency was mildly reduced. This is consistent with the typical lighter sleep noted in severe apneics.

Table 3 summarizes the Pearson correlation analyses. There were a number of statistically significant associations between various MFSI-sf subscales and sleep architecture measures. Physical fatigue scores were significantly associated with percent stage 1 and REM sleep ( $r=.329, p=.03, r=0.324, r=.04$, respectively), emotional fatigue with percent stage 1 sleep $(r=.329, p=.03)$, and total fatigue scores with percent stage 1 sleep $(r=.274$, $p=.02$ ). There were also a number of significant associations of fatigue scores with various arousal indices: including physical fatigue with total arousals $(r=0.360$, $p=.003)$, emotional fatigue with spontaneous movement arousals $(r=0.317, p=.009)$, emotional fatigue with total arousals $(r=0.416, p=0.001)$, and total MFSI-sf scores with total arousal scores $(r=0.269, p=0.03)$.

To control for multiple comparisons, we employed a MANOVA with MFSI-sf subscales as dependent variables and with sleep variables used in Table 3 as covariates. An ominibus $F$ test was obtained for each dependent variable; the models were significant for physical fatigue $(F=3.565, p=.001)$, emotional fatigue 
Table 3 Univariate correlations

Pearson's $r ; n=73$

${ }^{*} p<0.05, * * p<0.01$

\begin{tabular}{lllll}
\hline \multicolumn{2}{l}{ MFSI-sf Subscales } & & \\
\cline { 2 - 5 } & Physical & Emotional & Mental & Total \\
\hline Stage 1 sleep, \% & $.329^{* *}$ & $.353^{* *}$ & .065 & $.274^{*}$ \\
Stage 2 sleep, \% & .074 & .220 & .136 & .136 \\
Slow wave sleep, \% & .065 & .048 & .138 & .010 \\
REM sleep, \% & $.324^{* *}$ & .217 & $.255^{*}$ & .188 \\
Sleep efficiency, \% & .099 & .171 & .135 & .102 \\
WASO & .133 & .161 & $.252^{*}$ & .132 \\
AHI & $.331^{* *}$ & $.257^{*}$ & .088 & .227 \\
ODI & .241 & $.245^{*}$ & .005 & .194 \\
Total arousals & $.360^{* *}$ & $.416^{* *}$ & .085 & $.269^{*}$ \\
Snore arousals & .002 & .122 & .006 & .003 \\
Spontaneous cortical arousals & .111 & .046 & .145 & .007 \\
Respiratory cortical arousals & .145 & .061 & .017 & .140 \\
Spontaneous movement arousals & .107 & $.317^{* *}$ & .202 & .216 \\
Respiratory movement arousals & $.304^{*}$ & $.346^{* *}$ & .041 & .220 \\
Limb movement arousals & .169 & .056 & .056 & .049 \\
Periodic limb movement arousals & .241 & .137 & .106 & .086 \\
\hline
\end{tabular}

$(F=3.320, p=.001)$ and MFSI-sf total scores $(F=2.410$, $p=.014)$, but not MFSI-sf mental fatigue scores. Percent of stage 1 and REM sleep, as well as total arousals and movement arousals (spontaneous and respiratory) remained statistically significant $(p<.05)$ in the MANOVA analyses when we tested individual hypotheses and were then included in the linear regression modeling.

The results of the hierarchical linear regression models for each arousal index are shown in Tables 4, 5, and 6. For the MFSI-sf physical fatigue subscale and the MFSI-sf total scores, the final models were statistically significant, although percent stage 1 sleep, percent REM sleep, respiratory movement arousals, or total arousals did not independently contribute to variance of the dependent variables. For the MFSI-sf emotional fatigue subscale, the overall model was significant $(p<0.001)$ and while percent stage 1 sleep was not statistically significant, spontaneous movement arousals accounted for a significant proportion
Table 4 Multiple regression predictors of MFSI-sf emotional fatigue

\footnotetext{
${ }^{\text {a }} F(2,63)=6.96, p=0.002, R^{2}$

change 0.181

${ }^{\mathrm{b}} F(3,62)=27.64, p<0.001, R^{2}$

change 0.391

${ }^{\mathrm{c}} F(5,60)=16.42, \quad p<0.001, R^{2}$ change 0.006

${ }^{\mathrm{d}} F(8,57)=12.74, p<0.001, R^{2}$ change 0.064
}

\begin{tabular}{|c|c|c|c|c|c|c|}
\hline & $R$ & $R^{2}$ & Adjusted $R^{2}$ & Variables & $\beta$ & $P$ value \\
\hline \multirow[t]{2}{*}{ Step $1^{\mathrm{a}}$} & \multirow[t]{2}{*}{0.425} & \multirow[t]{2}{*}{0.181} & \multirow[t]{2}{*}{0.155} & Age & -0.001 & 0.991 \\
\hline & & & & BMI & 0.425 & 0.001 \\
\hline \multirow[t]{3}{*}{ Step $2^{\mathrm{b}}$} & \multirow[t]{3}{*}{0.756} & \multirow[t]{3}{*}{0.572} & \multirow[t]{3}{*}{0.551} & Age & 0.085 & 0.341 \\
\hline & & & & BMI & 0.219 & 0.020 \\
\hline & & & & CESD & 0.672 & $<0.001$ \\
\hline \multirow[t]{5}{*}{ Step $3^{\mathrm{c}}$} & \multirow[t]{5}{*}{0.760} & \multirow[t]{5}{*}{0.578} & \multirow[t]{5}{*}{0.543} & Age & 0.073 & 0.428 \\
\hline & & & & BMI & 0.195 & 0.106 \\
\hline & & & & CESD & 0.665 & $<0.001$ \\
\hline & & & & AHI & 0.000 & 0.998 \\
\hline & & & & ODI & 0.079 & 0.375 \\
\hline \multirow[t]{8}{*}{ Step $4^{\mathrm{d}}$} & \multirow[t]{8}{*}{0.801} & \multirow[t]{8}{*}{0.641} & \multirow[t]{8}{*}{0.591} & Age & 0.139 & 0.133 \\
\hline & & & & BMI & 0.276 & 0.021 \\
\hline & & & & CESD & 0.628 & $<0.001$ \\
\hline & & & & AHI & -0.102 & 0.645 \\
\hline & & & & ODI & 0.048 & 0.628 \\
\hline & & & & $\%$ Stage 1 sleep & -0.074 & 0.562 \\
\hline & & & & Spont movement & 0.326 & 0.003 \\
\hline & & & & Resp movement & 0.356 & 0.154 \\
\hline
\end{tabular}


Table 5 Multiple regression predictors of MFSI-sf physical fatigue
${ }^{\mathrm{a}} F(2,63)=15.681, p<0.001, R^{2}$ change 0.332

${ }^{\mathrm{b}} F(3,62)=16.312, p<0.001, R^{2}$

change 0.109

${ }^{\mathrm{c}} F(5,60)=9.537, \quad p<0.001, R^{2}$ change 0.002

${ }^{\mathrm{d}} F(8,57)=6.299, \quad p<0.001, R^{2}$ change 0.026

\begin{tabular}{|c|c|c|c|c|c|c|}
\hline & $R$ & $R^{2}$ & Adjusted $R^{2}$ & Variables & $\beta$ & $P$ value \\
\hline \multirow[t]{2}{*}{ Step $1^{\mathrm{a}}$} & 0.577 & 0.332 & 0.311 & Age & 0.111 & 0.311 \\
\hline & & & & BMI & 0.602 & $<0.001$ \\
\hline \multirow[t]{3}{*}{ Step $2^{b}$} & 0.664 & 0.441 & 0.414 & Age & 0.156 & 0.127 \\
\hline & & & & BMI & 0.494 & $<0.001$ \\
\hline & & & & CESD & 0.355 & 0.001 \\
\hline \multirow[t]{5}{*}{ Step $3^{\mathrm{c}}$} & 0.665 & 0.443 & 0.396 & Age & 0.148 & 0.166 \\
\hline & & & & BMI & 0.492 & 0.001 \\
\hline & & & & CESD & 0.351 & 0.001 \\
\hline & & & & AHI & -0.018 & 0.887 \\
\hline & & & & ODI & 0.040 & 0.695 \\
\hline \multirow[t]{8}{*}{ Step $4^{\mathrm{d}}$} & 0.685 & 0.469 & 0.395 & Age & 0.137 & 0.218 \\
\hline & & & & BMI & 0.438 & 0.003 \\
\hline & & & & CESD & 0.373 & 0.002 \\
\hline & & & & AHI & 0.230 & 0.395 \\
\hline & & & & ODI & 0.088 & 0.427 \\
\hline & & & & $\%$ Stage 1 sleep & 0.030 & 0.848 \\
\hline & & & & \% REM sleep & -0.133 & 0.236 \\
\hline & & & & Resp movement & -0.334 & 0.261 \\
\hline
\end{tabular}

of the variance of the MFSI-sf emotional fatigue scores $\left(R^{2}\right.$ change 0.06, $p=0.001)$. Depression scores and BMI contributed significantly to the variance of the physical and MFSI-sf total score fatigue models. None of the demographic or sleep apnea severity characteristics contributed significantly towards the fatigue scores. Mental fatigue scores were not evaluated with hierarchical regression because the MANOVA model was not statistically significant for mental fatigue.

\section{Discussion}

This study sought to investigate the roles of sleep architecture and arousal frequency in patients with OSA, particularly the potential relationship of sleep fragmentation with fatigue. A central finding of this study was that amounts of stage 1 and REM sleep, as well as various arousal indices, were associated with increased fatigue scores. After controlling for potential contributors to fatigue
Table 6 Multiple regression predictors of MFSI-sf total scores

\begin{tabular}{|c|c|c|c|c|c|c|}
\hline & $R$ & $R^{2}$ & Adjusted $R^{2}$ & Variables & $\beta$ & $P$ value \\
\hline \multirow[t]{2}{*}{ Step $1^{\mathrm{a}}$} & \multirow[t]{2}{*}{0.497} & \multirow[t]{2}{*}{0.247} & \multirow[t]{2}{*}{0.222} & Age & 0.191 & 0.106 \\
\hline & & & & BMI & 0.525 & $<0.001$ \\
\hline \multirow[t]{3}{*}{ Step $2^{\mathrm{b}}$} & \multirow[t]{3}{*}{0.724} & \multirow[t]{3}{*}{0.524} & \multirow[t]{3}{*}{0.501} & Age & 0.265 & 0.007 \\
\hline & & & & BMI & 0.355 & 0.001 \\
\hline & & & & CESD & 0.565 & $<0.001$ \\
\hline \multirow[t]{5}{*}{ Step $3^{\mathrm{c}}$} & \multirow[t]{5}{*}{0.725} & \multirow[t]{5}{*}{0.525} & \multirow[t]{5}{*}{0.485} & Age & 0.258 & 0.011 \\
\hline & & & & BMI & 0.386 & 0.003 \\
\hline & & & & CESD & 0.565 & $<0.001$ \\
\hline & & & & AHI & -0.047 & 0.693 \\
\hline & & & & ODI & -0.008 & 0.933 \\
\hline \multirow[t]{7}{*}{ Step $4^{\mathrm{d}}$} & \multirow[t]{7}{*}{0.730} & \multirow[t]{7}{*}{0.532} & \multirow[t]{7}{*}{0.475} & Age & 0.264 & 0.014 \\
\hline & & & & BMI & 0.379 & \\
\hline & & & & CESD & 0.603 & 0.005 \\
\hline & & & & AHI & 0.050 & $<0.001$ \\
\hline & & & & ODI & 0.018 & 0.805 \\
\hline & & & & $\%$ Stage 1 Sleep & -0.085 & 0.856 \\
\hline & & & & Total Arousals & -0.138 & 0.556 \\
\hline
\end{tabular}


such as demographic information, depression, and sleep apnea severity, hierarchical linear regression analyses demonstrated that spontaneous movement arousals contributed significantly to the variance of emotional fatigue scores.

This association of arousals with fatigue is interesting. While fatigue is a common symptom in patients with OSA, little is known about what drives it. Prior investigations into sleep apnea severity, particularly the apnea hyoponea index or measures of oxygen desaturation, have not shown significant associations with fatigue scores [14, 15]. Depression has been demonstrated to be a significant independent contributor to fatigue in patients with OSA but is unlikely to be the sole causative entity [15]. There is limited information about fatigue and arousals in patients with OSA, although there are some preliminary studies in other patient populations. A study of patients with multiple sclerosis found significantly increased self reports of fatigue and poor sleep quality. The polysomnograms for such patients revealed increased amounts of sleep fragmentation and arousals [30]. In a group of subjects with high levels of occupational stress and mental fatigue, disturbances in sleep architecture (more stage 1 and decreased slow wave and REM sleep) and increased arousal frequency were observed [31].

Our finding that movement arousals made particularly significant contributions to emotional fatigue scores is important, as it is one of the first reports of a potentially new etiologic factor in the fatigue of apneics. The finding that a specific type of arousal (spontaneous movement) was particularly associated with emotional fatigue is also interesting and unexpected. Although disturbances in polysomnographic variables and increased arousals were positively correlated with physical and total fatigue scores, these variables were not statistically significant after controlling for other factors that could be contributing to fatigue scores.

It is also interesting to note that emotional fatigue scores were the subtype most influenced by arousal severity. The MFSI items that contribute to the emotional fatigue scores focus heavily on mood disruption and anxiety. One can speculate that the emotional fatigue subscale of the MFSI-sf measures fatigue associated with depressive symptoms and that movement arousals may influence fatigue through or in concert with changes in depressive symptoms. In this study, depression scores contributed a significant amount of variance to fatigue scores as well as movement arousals. Given that this is a cross-sectional study, it is impossible to fully delineate the relationship between arousals, fatigue, and depression. However, it is likely that depression plays an important role in the perceived symptoms of patients with OSA. Prior studies have found disproportionate incidence of depression in patients with OSA [32, 33].
When patients were treated for their OSA, depression scores were noted to improve [32]. Overall, this is suggestive of a psychological component to the fatigue that apneic patients experience.

Limitations of our study include our sample size and our multiple Pearson correlation comparisons. We felt it was relevant to perform a first pass screen of relevant sleep architecture variables and arousal indices with fatigue measures, as there is limited literature that supports any specific variable over another. Each polysomnographic parameter also likely represents a physiologically distinct variable and we felt each deserved attention in our analyses. As a check against multiple comparisons, we employed a MANOVA approach that tested overall models of fatigue with sleep parameters as covariates. The MANOVA was statistically significant for three of the models examined, with the exception of the mental fatigue measures, which were not included in the final analyses. We also only included variables in our linear regression modeling that were noted to be significant in the MANOVA analyses. The hierarchical linear regression served as another form of statistical control of our multiple comparisons, as it showed significance primarily with movement arousals, after controlling for a number of potential contributors to fatigue scores.

Although we noted positive associations of fatigue with various sleep measures (stage 1 sleep, AHI, and total arousals), these measures are not necessarily independent measures; all of these measures can be potential markers of sleep apnea severity. We included these parameters in our hierarchical linear regression to evaluate contributions to fatigue and as noted previously, only movement arousals were statistically significant. The lack of significance of the other parameters such as stage 1 sleep and AHI in the final model suggests that although they may be related to fatigue, their relationship is not sufficiently strong to make them significant contributors in the final hierarchical linear model, after controlling for arousals.

In this study, we have examined total arousals in the context of cortical and movement arousals and further subdivided them into those of spontaneous and respiratory origin. Although one would not necessarily expect to see different types of arousals causing differential responses on fatigue, we have previously found some utility in examining specific types of arousals. For instance, in a prior study of patients with obstructive sleep apnea, it was noted that movement arousals were associated with increased sympathetic nervous system functioning but cortical arousals were not [21]. Given the potential for an increased contribution of movement arousals on outcomes in patients with OSA, we believe that further investigation of subtypes of arousals is indicated.

Our study population is quite homogenous and "healthy" for an OSA sample as we excluded any patients with other 
chronic medical conditions. It is possible that other disease processes commonly associated with OSA, such as diabetes mellitus, are important etiologic factors in their fatigue as well. Another potential limitation of our study is that our participants may have confused symptoms of sleepiness with fatigue. We attempted to minimize this confusion by using an instrument that has been validated for use in measuring fatigue in different populations. Although sleepiness and fatigue are often unfortunately used interchangeably by clinicians, studies have shown that patients are able to identify each as a distinct symptom [3-5].

Although we presented data showing significant correlations of spontaneous movement arousals with fatigue, these arousals were a relatively uncommon occurrence in our sample population. The clinical significance of our findings remains to be determined. Might it be useful in the future to target arousals in order to treat fatigue? This study provides an early report of a positive association between fatigue and movement arousals, a finding not previously noted in the literature.

In conclusion, we presented evidence for a potential role of sleep fragmentation in the fatigue seen with obstructive sleep apnea, through changes in sleep architecture and increased arousals. We also provided evidence that movement arousals contributed a significant amount to emotional fatigue scores.

Acknowledgements This work was supported by grants HL44915, M01 RR00827, CA23100, AG08415, and CA112035 from the National Institutes of Health.

Open Access This article is distributed under the terms of the Creative Commons Attribution Noncommercial License which permits any noncommercial use, distribution, and reproduction in any medium, provided the original author(s) and source are credited.

\section{References}

1. Young T, Skatrud J, Peppard PE (2004) Risk factors for obstructive sleep apnea in adults. JAMA 291:2013-2016. doi:10.1001/jama.291.16.2013

2. Young T, Palta M, Dempsey J, Skatrud J, Weber S, Badr S (1993) The occurrence of sleep-disordered breathing among middle-aged adults. N Engl J Med 328:1230-1235. doi:10.1056/ NEJM199304293281704

3. Hossain JL, Ahmad P, Reinish LW, Kayumov L, Hossain NK, Shapiro CM (2005) Subjective fatigue and subjective sleepiness: two independent consequences of sleep disorders? J Sleep Res 14:245-253. doi:10.1111/j.1365-2869.2005.00466.x

4. Chervin RD (2000) Sleepiness, fatigue, tiredness, and lack of energy in obstructive sleep apnea. Chest 118:372-379. doi:10.1378/chest.118.2.372

5. Aguillard RN, Reidel BW, Lichstein KL, Grieve FG, Johnson CT, Noe SL (1998) Daytime functioning in obstructive sleep apnea patients: exercise tolerance, subjective fatigue, and sleepiness. Appl Psychophysiol Biofeedback 23:207-217. doi:10.1023/ A:1022257514209
6. Shen J, Barbera J, Shapiro JM (2006) Distinguishing sleepiness and fatigue: focus on definition and measurement. Sleep Med Rev 10:63-76. doi:10.1016/j.smrv.2005.05.004

7. Dimsdale JE, Ancoli-Israel S, Elsmore TF, Gruen W (2003) Taking fatigue seriously: I. Variations in fatigue sampled repeatedly in healthy controls. J Med Eng Technol 27:218-222. doi:10.1080/0309190031000075354

8. Kroenke K, Price RK (1993) Symptoms in the community, prevalence, classification, and psychiatric comorbidity. Arch Intern Med 153:2474-2480. doi:10.1001/archinte.153.21.2474

9. Ream E, Richardson A (1997) Fatigue in patients with cancer and chronic obstructive airways disease: a phenomenological enquiry. Int J Nurs Stud 34:44-53. doi:10.1016/S0020-7489 (96)00032-6

10. Krupp LB, La Rocca NG, Muir-Nash J et al (1989) The fatigue severity scale: application to patients with multiple sclerosis and systemic lupus erythematosus. Arch Neurol 46:1121-1123

11. Vogelzang NJ, Breitbart W, Cella C, Curt GA, Groopman JE, Horning SJ, Itri LM, Johnson DH, Scherr SL, Protenoy RK (1197) Patient, caregiver, and oncologist perceptions of cancer-related fatigue: results of a tri-part assessment survey. Sem Hematology 13:4-12

12. Kribbs NB, Pack AI, Kline LR, Getsy JE, Schuett JS, Henry JN, Maislin G, Dinges DF (1993) Effects of one night without nasal CPAP treatment on sleep and sleepiness in patients with obstructive sleep apnea. Am Rev Respir Dis 147:1162-1168

13. Watson RK, Thompson AS (1992) Treatment outcome of sleep apnea. Conn Med 56:125-129

14. Lichstein KL, Means MK, Noe SL, Aguillard RN (1997) Fatigue and sleep disorders. Behav Res Ther 35:733-740. doi:10.1016/ S0005-7967(97)00029-6

15. Bardwell WA, Moore P, Ancoli-Israel S, Dimsdale JE (2003) Fatigue in obstructive sleep apnea: driven by depressive symptoms instead of apnea severity? Am J Psychiatry 160:350-355. doi:10.1176/appi.ajp. 160.2.350

16. Bardwell WA, Ancoli-Israel S, Dimsdale JE (2007) Comparison of the effects of depressive symptoms and apnea severity on fatigue in patients with obstructive sleep apnea: a replication study. J Affect Disord 97:181-186. doi:10.1016/j.jad.2006.06.013

17. Guilleminault C, Poyares D, da Rosa A, Kirisoglu C, Almeida T, Lopes MC (2006) Chronic fatigue, unrefreshing sleep, and nocturnal polysomnography. Sleep Med 7:513-520. doi:10.1016/ j.sleep. 2006.03.016

18. Reeves WC, Heim C, Maloney EM, Youngblood LS, Unger ER, Decker MJ, Jones JF, Rye DB (2006) Sleep Characteristics of persons with chronic fatigue syndrome and non-fatigued controls: results from a population based study. BMC Neurol 16:41-49. doi:10.1186/1471-2377-6-41

19. Thomas KS, Bardwell WA, Ancoli-Israel S, Dimsdale JE (2006) The toll of ethnic discrimination on sleep architecture and fatigue. Health Psychol 25:635-642. doi:10.1037/0278-6133.25.5.635

20. Elmenhorst EM, Elmenhorst D, Luks N, Maass H, Vejvoda M, Samel A (2007) Partial Sleep deprivation: Impact on the architecture and quality of sleep. Sleep Med; Epub ahead of print.

21. Loredo JS, Ziegler MG, Ancoli-Israel S, Clausen JL, Dimsdale JE (1999) Relationship of arousals from sleep to sympathetic nervous system activity and BP in obstructive sleep apnea. Chest 116:655656. doi:10.1378/chest.116.3.655

22. Guilleminault C, Lopes MC, Hagen CC, da Rosa A (2007) The cyclic alternating pattern demonstrates increased sleep instability and correlates with fatigue and sleepiness in adults with upper airway resistance syndrome. Sleep 30:641-647

23. Radloff LS (1977) The CES-D Scale: a self report depression scale for research in the general population. J Appl Psychol Meas 1:385-401. doi:10.1177/014662167700100306 
24. Stein KD, Martin SC, Hann DM, Jacobsen PB (1998) A multidimensional measure of fatigue for use with cancer patients. Cancer Pract 6:143-152. doi:10.1046/j.15235394.1998.006003143.x

25. Stein KD, Jacobsen PB, Blanchard CM, Thors C (2004) Further validation of the multidimensional fatigue symptom inventoryshort form. J Pain Symptom Manage 27:14-23. doi:10.1016/j. jpainsymman.2003.06.003

26. Rechtshaffen A, Kales A (1968) A manual of standardized terminology, techniques, and scoring system for sleep stages of human subjects. National Institute of Health Publication 204: US Government Printing Office Washington, DC

27. Report ASDA (1992) EEG arousals: scoring rules and examples. Sleep 15:174-184

28. Loredo JS, Clausen JL, Ancoli-Israel S, Dimsdale JE (1999) Night-to-night arousal variability and interscorer reliability of arousal measurements. Sleep 22:916-920
29. Miller RG (1980) Simultaneous statistical inference: Springer series in statistics. Springer-Verlag Press, New York

30. Kaynak H, Altintaș A, Kaynak D, Uyanik O, Saip S, Ağaoğlu J, Onder G, Siva A (2006) Fatigue and sleep disturbance in multiple sclerosis. Eur J Neurol 13:1333-1339. doi:10.1111/j.14681331.2006.01499.x

31. Ekstedt M, Söderström M, Akerstedt T, Nilsson J, Søndergaard HP, Aleksander P (2006) Disturbed sleep and fatigue in occupational burnout. Scand J Work Environ Health 32:121-131

32. Schwartz DJ, Kohler WC, Karatinos G (2005) Symptoms of depression in individuals with obstructive sleep apnea may be amenable to treatment with continuous positive airway pressure. Chest 128:1304-1309. doi:10.1378/chest.128.3.1304

33. Wells RD, Day RC, Carney RM, Freedland KE, Duntley SP (2004) Depression predicts self-reported sleep quality in patients with obstructive sleep apnea. Psychosom Med 66:692-697. doi:10.1097/01.psy.0000140002.84288.e1 